\title{
Canadian women opting for less effective birth control
}

Cite as: CMAJ 2017 July 10;189:E921-2. doi: 10.1503/cmaj.1095446

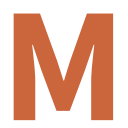

ore Canadian women are relying on less effective methods of birth control and are using them less consistently than they did a decade ago, according to the Society of Obstetricians and Gynaecologists of Canada (SOGC).

The SOGC surveyed more than 3200 Canadian women aged 15 to 50 about their awareness and use of contraceptive methods, first in 2006 and again in 2016. The latest results, presented June 21 at the SOGC's annual conference in Ottawa, show there's been little progress and a possible backlash against oral contraceptives in recent years.

Condoms, the pill and "pulling out" are still the most popular methods of birth control among Canadian women. However, oral contraceptive use dropped among women over 30 from $39 \%$ in 2006 to $15.7 \%$ in 2016 . Meanwhile, reliance on condoms alone increased across all age groups, with a marked uptick among teens from $21 \%$ to $50.1 \%$.

"It's concerning that we're still using methods of contraception that have a higher failure rate with typical use when we have other more effective methods available," like intrauterine devices (IUDs), says Dr. Amanda Black of the SOGC's Contraception Awareness Program Working Group.

Although four out of five women use some form of birth control at least some of the time, one in four uses those methods inconsistently, "more so than we'd seen in the previous survey," says Black. More worrying is the increasing number of women under 30 who don't use any form of contraception. Nearly one in 10 sexually active teens surveyed in 2016 said they do nothing to prevent pregnancy, up from less than one in 20 in 2006. Among women in their $20 \mathrm{~s}, 12.5 \%$ of single women and $22.3 \%$ of those who are married or in common law relationships were not using any method of birth control, up from $9.1 \%$ and $19.3 \%$ respectively.

Not surprisingly, "the number of women surveyed who reported an unintended pregnancy was essentially unchanged," says Black. One in five Canadian women had an unplanned pregnancy in 2016, the same as in 2006. One in three of these women had abortions, up from one in four a decade earlier. The average number of unintended pregnancies also increased slightly, from 1.58 to 1.84 among single women and 1.55 to
1.69 among those married or common law.

Black says the results are a wake-up call for women's health providers. "If things haven't changed in 10 years, what are we doing wrong and how can we do things better?"

Contraception misuse or failure appears to be a factor, since almost half pregnancies in 2016 were using some method of birth control, compared to less than a third of women the decade before.

There also seems to be a perfect storm of ignorance about contraceptive options, increasing concern about adverse effects the women who reported unintended

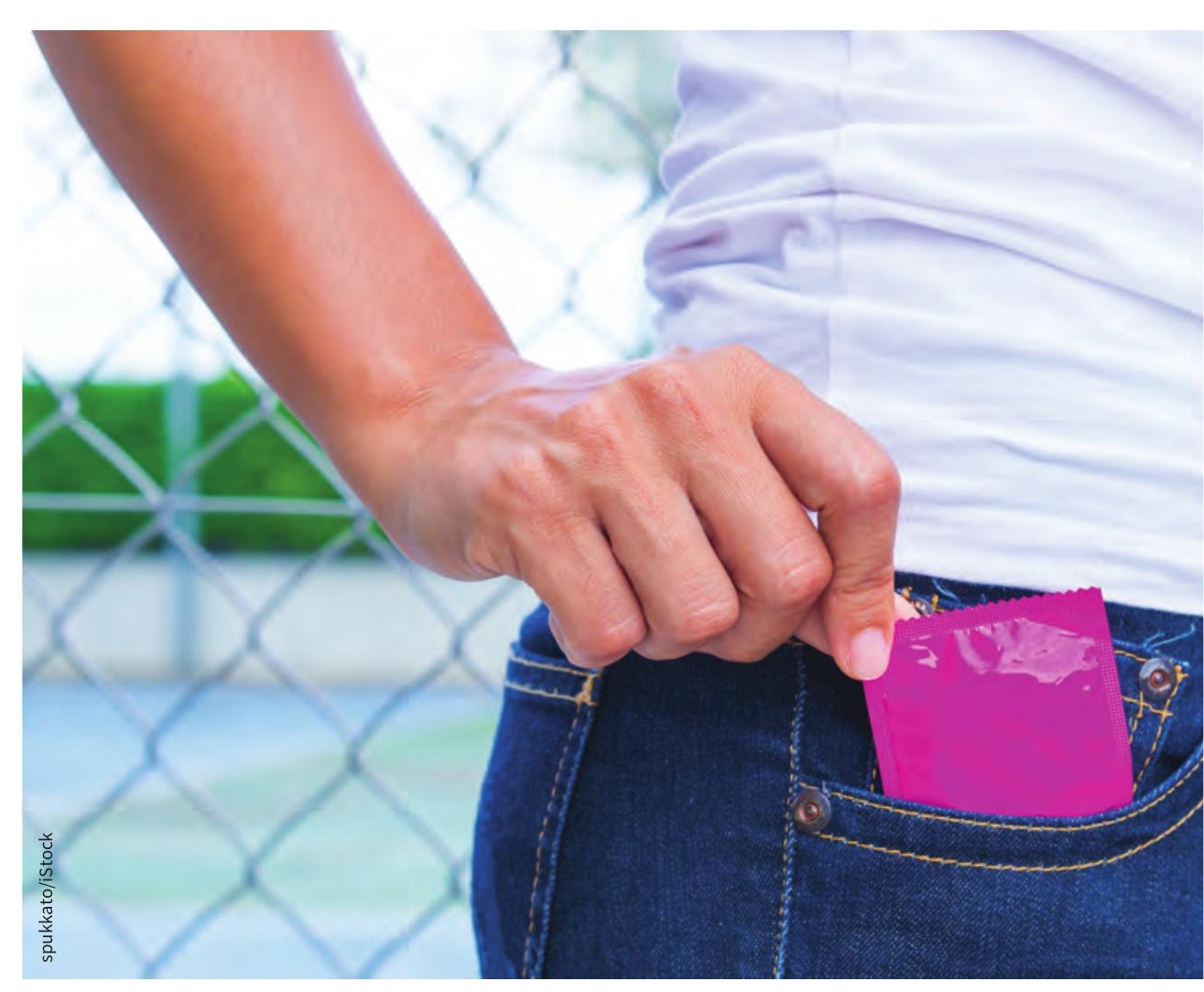

Canadian women are ditching the pill in favour of condoms, or sometimes even less reliable contra-

ception, according to a national survey. 
and eroding trust in health providers as more women seek advice online.

"There's still a lot of misinformation out there which may limit women's ability to choose a contraceptive method that's right for them," explains Black.

The SOGC tested women's awareness of 24 types of contraception, including forms of sterilization, hormonal birth control, device and barrier methods, and "natural" options like breastfeeding, withdrawal, abstinence and the rhythm method. Most women were unaware of more than half of these methods, including some of the most effective options such as IUDs and contraceptive implants. In fact, less than a quarter of women thought hormonal IUDs were "very effective," while fewer than one in five said the same about contraceptive implants.

Women also underestimated their chances of getting pregnant. More than a third of those who had unplanned pregnancies said they didn't use contraception at the time because they didn't think they could get pregnant. Notably, $27 \%$ said they were unprepared in the heat of the moment and $17.8 \%$ said they didn't use contraceptives because of the adverse effects.

"It's common for women to take pill breaks or discontinue birth control assuming they will have time to restart it," says Dr. Dustin Costescu, a family planning specialist at McMaster University in Hamilton. Health providers often recommend breaks when women complain of contraceptive adverse effects "with no discussion about what else could be used in the interim," Costescu says.

Fifty-one percent of women using hormonal birth control reported adverse effects in 2016, up from $46.8 \%$. The most common adverse effects - weight gain and headaches - were also listed among the top reasons for discontinuing a current method of contraception. But instead of switching to more effective alternatives, a quarter of women who stopped birth control for any reason turned to withdrawal or had unprotected sex.

How doctors counsel their patients about contraceptives may be the crux of the problem. Family doctors are still the top source of contraceptive information, but only $58.8 \%$ of women turn to them for advice, down from $71.3 \%$ in 2006 . More troubling, only $54.2 \%$ consider them trustworthy.

Meanwhile, the internet has become the second most used source of information, with half of women seeking advice online up from a quarter in 2006. Although only one in five women trust the information they find online, they use and trust sexual health clinics, pharmacists and nurses even less.

The reason for this shift is a simple one, says Laura Wershler, a Calgarybased women's health advocate and member of the Society for Menstrual Health Research. "Women have not felt heard," particularly when it comes to their concerns about the safety and adverse effects of contraceptives, or their desire to use natural alternatives, she says. Doctors tend to default to prescribing oral contraceptives or other hormonal contraception, "but they do a poor job of acknowledging and hearing young women who say they don't want to do that."

For some women, " $100 \%$ pregnancy prevention isn't the be all and end all," Wershler adds. "Physicians and sexual health clinics should be honoring these women's choices."

Costescu argues that physicians who are dismissive of less effective methods may be setting up their patients for contraceptive mishap. "Women who dislike their contraceptive care providers are more likely to experience a failure or discontinuation of their method, so quality of counselling is very important."

That may mean taking more time to understand, rather than change a patient's perspective, and instead of steering her away from certain methods, supporting her to use them as effectively as possible, he explains.

Black says that it may be time to rethink the primacy of the pill in these discussions. "Women are concerned and rightly because there have been issues with safety in the past," she says. "Probably we need to flip things around and talk about methods starting at the most effective and work our way down."

\section{Lauren Vogel, CMAJ}

